404

\section{COMPARISON OF BODY FAT ESTIMATION USING SKIN FOLD THICKNESS \\ MEASUREMENT AND SIMULTANEOUS AIR- DISPLACEMENT PLETHYSMOGRAPHY AT 8 WEEKS}

N. Hou Liu' ${ }^{1}$, J.O. Hourihane ${ }^{2}$, L. Kenny ${ }^{3}$, M. Kiely ${ }^{4}$, A.D. Irvine ${ }^{5}$, D.M. Murray ${ }^{2}$

${ }^{1}$ University College Cork, ${ }^{2}$ Paediatrics and Child Health, ${ }^{3}$ Obstetrics and Gynaecology, ${ }^{4}$ Food

Science and Nutrition, University College Cork, ${ }^{5}$ Dermatology, Trinity College Dublin, Cork, Ireland

Background and aims: There is increasing interest in early life body composition as a predictor of later obesity and metabolic dysfunction. Air displacement plethysmography offers direct measurement of body composition in infants. We wished to examine the reliability of traditional skin fold thickness measurements to estimate body fat composition at 8 weeks of age.

Methods: Infants were recruited antenatally as part of a prospective birth cohort study, the BASELINE study. Body composition was assessed at 8 weeks of life in infants using air displacement body plethesmography $\left(\% \mathrm{BF}_{\mathrm{ADP}}\right)$. Skin fold thickness $\left(\% \mathrm{BF}_{\mathrm{SFT}}\right)$ was measured a triceps and subscapular regions using a Holtain callipers. Body fat was estimated from these readings using the sex specific equations: Males: $\% B F=1.21 \mathrm{x}$ $\Sigma$ SFT $-0.008 \times(\Sigma S F T)^{2}-1.7$. Females: $\% B F=$ $1.33 \times \sum$ SFT $-0.013 \times\left(\sum \mathrm{SFT}\right)^{2}-2.5\left(\sum \mathrm{SFT}=\right.$ sum of triceps and subscapular skinfold thickness measurements)Results: Data were collected from 151 infants; mean (SD) gestation=39.6 (1.5), birth weight $=3480(503)$ grams. The mean (SD) body fat $=21.07(4.01 \%)$ using $\% \mathrm{BF}_{\mathrm{ADP}}$ and $11.49(2.20) \%$ using $\% \mathrm{BF}_{\mathrm{SFT}}$. $\% \mathrm{BF}_{\mathrm{SFT}}$ consistently underestimated body fat. The mean (SD) difference between the 2 methods of measurement was $9.58(0.29) \%$. This mean (SD) difference increased significantly as $\% \mathrm{BF}_{\mathrm{ADP}}$ increased [5.5(2.4) lowest quartile $\% \mathrm{BF}_{\mathrm{ADP}}$ vs 13.01(2.10) highest quartile $\left.\% \mathrm{BF}_{A D P} p<0.0005\right]$.

Conclusion: We have provided references ranges for infant fat mass at 8 weeks of life. Skin fold thickness calculations underestimate body fat, particularly in infants with the highest body fat $\%$.
405

\section{CARDIOVASCULAR RISK FACTORS IN CHILDREN WITH TYPE 1 DIABETES: A CROSS- SECTIONAL STUDY OF A MIXED FLEMISH- MAGHREB POPULATION IN BRUSSELS}

\author{
W. Dewals, I. Gies, J. Vanbesien, J. De Schepper
}

Universitair Kinderziekenhuis Brussel, Jette, Belgium

Background: Traditional risk factors for cardiovascular disease such as an abnormal lipid profile, hypertension, obesity, and insulin resistance contribute to the accelerated atherosclerosis seen in young persons with T1D. Gender, ethnic origin, pubertal status and metabolic control might influence these parameters during childhood.

Aims: To compare the prevalence of dyslipidemia, hypertension as well as global and abdominal obesity between Flemish and Maghreb children receiving similar standard medical care and to analyze the relationship of these parameters with serum hS CRP and fibrinogen, as parameters of a general state of inflammation, which can be considered as a precursor of atherosclerosis.

Patients and methods: At their annual check up, fasting lipid levels, hS CRP, fibrinogen, blood pressure, body weight and length as well as waist circumference were determined in children and adolescents with T1DM at least 1 year after diagnosis and without familial hypercholesterolemia or treatment with statins. Anthropometric data were expressed as z-scores for age using recent Flemish population references (2004). ISPAD defined target levels (2009) for lipid levels were used.

Conclusion: Type 1 diabetes children from Maghreb origin have a higher degree of obesity, but similar lipid levels than innate children.

Elevated LDL is the most frequently encountered cardiovascular risk factor using the ISPAD cut-offs

Inflammation as well as elevated LDL can already be observed in pre-pubertal children with type 1 diabetes, unrelated to their metabolic control.

$\mathrm{HbA1c}$ correlated significantly with diastolic blood pressure and serum triglycerides but also with parameters for subclinical atherosclerosis as fibrinogen and hs CRP. 